\title{
GRADED POLYNOMIAL IDENTITIES OF TRIANGULAR ALGEBRAS
}

\author{
ONOFRIO M. DI VINCENZO AND VINCENZO NARDOZZA ${ }^{凶}$
}

\begin{abstract}
Let $F$ be any field, $G$ a finite abelian group and let $A, B$ be $F$ algebras graded by subgroups of $G$. If $M$ is a $G$-graded free $(A, B)$-bimodule, we describe the $G$-graded polynomial identities of the triangular algebra of $M$ and, in case the field $F$ has characteristic zero, we provide the description of its $G$-graded cocharacters by means of the graded cocharacters of $A$ and $B$.
\end{abstract}

\section{INTRODUCTION}

Let $F$ be an arbitrary field and consider a pair of $F$-algebras $A, B$ graded by subgroups $H$ and $K$ of an abelian finite group $G$. For any $G$-graded $(A, B)$-bimodule $M$ the triangular algebra $R=\left(\begin{array}{cc}A & M \\ & B\end{array}\right)$ has a natural $G$-graded algebra structure.

In the present paper we determine the $G$-graded polynomial identities of $R$ (Theorem 10) provided that the graded components of $M$ are in some (specified) sense free and the $G$-supports of $M$ and $A \oplus B$ are disjoint. This result does not depend on the characteristic of the base field $F$ nor on its cardinality, so the results are valid for finite fields too. When $F$ has characteristic zero we provide the $S_{n}$-structure of the $G$-graded multilinear spaces $P_{n}^{G}(R)$, related to the $S_{n}$-cocharacters of $A, B$ and $A \oplus B$ (Theorem 17 and Corollary 19).

Triangular algebras are a well known device in emphasizing different left and right behaviors in non-commutative setups (see for instance the synthetic and yet agreeable digressions in [11], Example 1.14), but are also of interest in representation theory of algebras, most prominently due to a beautiful paper of Lewin [12]. His results may apply to problems pertaining PI-Theory of associative algebras, as it was shown in the same paper; actually Theorem 1 in [12] can be stated in a PIsetup, see [7] Corollary 1.8.2, and employed as an effective tool to compute the $T$-ideal of block-triangular matrices as in Section 4 of [8].

Lewin's Theorem has been indeed generalized, as in Section 3 of [5], to setups involving graded algebras and graded polynomial identities, a topic of greatly increasing interest during the past 20 years. The reason in such interest is probably twofold. In first place, graded algebras behaves well from several points of view: they are beautiful structures, with an elegant complexity manifesting in their graded polynomial identities, much nicer than the ordinary polynomial identities. The second reason is that graded algebras are an essential ingredient in studying ordinary polynomial identities of algebras. This became evident since Kemer's work [10],

2010 Mathematics Subject Classification. Primary 16R50, Secondary 16W50, 16W99.

Key words and phrases. Graded polynomial identities, graded algebra, graded cocharacters.

( $₫$ Corresponding Author) Partially supported by GNSAGA 2018

This is a post-print of the paper available at https:// doi.org/10.1016/j.jpaa.2019.106256. 
where $\mathbb{Z}_{2}$-graded algebras (also named superalgebras) play a key role in the representability of PI-algebras, as well as in the structure theory of $T$-ideals in terms of $T$-prime ideals. The pervading presence of $\mathbb{Z}_{2}$-graded algebras affects also the classification of varieties through the so called PI-exponent: every minimal variety is generated by a suitable $\mathbb{Z}_{2}$-graded algebra [9]. However the connections between graded and ordinary polynomial identities are present even at very basic level: it is well known and easy to prove that if two $G$-graded algebras $A$ and $B$ have the same graded polynomial identities then they must have the same ordinary polynomial identities.

Whatever weighted mix of these two reasons, or possibly for further ones, the investigations about graded algebras (associative, Lie, Jordan) received a great impulse, both in classifying the gradings of relevant algebras and in extending important ordinary (ungraded) theorems to graded settings. We must cite for instance that Kemer's representability theorem, the solution of Specht's problem and the Amitsur's conjecture on the existence of the PI-exponent have been restated for $G$-graded algebras [1], [13], [2].

In the present paper, we investigate the hidden backbone of several results involving block triangular matrices. In the typical setup, the $0_{G}$-component of $R$ is constituted by $A \oplus B$ while $M$ provides the other nonzero $G$-components of $R$. Thus the only nontrivial graded polynomial identities of $A$ and $B$ are the ordinary (non graded) ones. The easiest case is when $A=B=M=F$ and $A, B$ are trivially graded by a group $G$. Namely, this amounts to consider a non trivial $G$-grading on the algebra $U T_{2}(F)$, as in [14] (actually, in this case the results follow directly from [4]). The results in [4], describing the $\mathbb{Z}_{2}$-graded polynomial identities of $R$ when $A$ and $B$ are trivially graded and $M$ is the component of degree $1 \in \mathbb{Z}_{2}$, and those of [6], describing the $\mathbb{Z}_{2}$-graded cocharacters of $R$ in the same settings, constitute the motivating examples of the present paper. As it turns out, apart from some (strong) freeness condition on $M$, the very reason why they work relies not on the fact that $A$ and $B$ have a specific (trivial) grading, but on the fact that the $G$-supports of $A \oplus B$ and $M$ are disjoint. Hence all these results can be extended to a considerably much wider scope. Indeed, although far from being the most general case, this assumption is strong enough to provide effective general statements and computability. When the $G$-supports are not disjoint many entirely different situations may arise, and more specific assumptions are needed in order to get to conclusions. As an instance, in [5] the notion of $G$-regularity of $A$ or $B$ supplies the lack of disjointness of the supports and causes $T_{G}(R)$ being squeezed to $T_{G}(A) T_{G}(B)$, thus providing a graded version of the original result of Lewin. However $G$-regularity is a condition even stronger than support disjointness: in the general case, $T_{G}(R)$ is simply sandwiched between $T_{G}(A) T_{G}(B)$ and $T_{G}(A) \cap T_{G}(B)$.

The paper is sectioned in the following way: Section 2 collects the general definitions and results needed to study $G$-graded polynomial identities of an associative unitary $F$-algebra, in a tentative to keep the paper as self-contained as possible. A new formalization of the general results in [3], resulting in a more comfortable notation, is succinctly provided. The construction of the $G$-graded triangular algebra $R$ is contained in Section 3, together with some general remarks and the main assumptions on the problem we are going to deal with. The first new contribution appears in Section 4, devoted to determine the $G$-graded polynomial identities of $R$ in terms of the graded polynomial identities of $A$ and $B$. Finally Section 5 deals 
with the $S_{n}$-structure of the graded multilinear polynomial identities of $R$ through the representation theory of the symmetric groups, in case the field $F$ has characteristic zero. Precisely, in Theorem 17 we describe the $S_{n}$-structure of $P_{n}^{G}(R)$ and in Corollary 19 we provide a simple formula relating it to those determined by $A$ and $B$.

\section{BASIC NOTIONS AND NOTATION}

This section is devoted to a very quick survey on the main definitions, notations and results used in the rest of the paper. For the moment, assume that $F$ is any (possibly finite) field. By $F$-algebra we mean an associative unitary algebra over the base field $F$. If $(G,+)$ is any group, an $F$-algebra $A$ is $G$-graded if it admits a direct decomposition into $F$-subspaces indexed by $G, A=\bigoplus_{g \in G} A_{g}$, satisfying $A_{g} A_{h} \subseteq A_{g+h}$ for all $g, h \in G$. The additive notation for the operation in $G$ is consistent with our setup since in this paper we will be concerned with abelian groups only. The subspace $A_{g}$ is called the $g$-homogeneous component of $A$, and an element $a \in A$ is $G$-homogeneous of degree $g$ if $a \in A_{g}$. If $A, B$ are $G$-graded algebras, a $G$-homomorphism is any algebra homomorphism $\varphi: A \rightarrow G$ preserving the homogeneous components, that is $\varphi\left(A_{g}\right) \subseteq B_{g}$ for all $g \in G$.

A free object for the class of $G$-graded algebras can be naturally defined: consider the set $X_{G}:=\left\{x_{i, g} \mid 1 \leqslant i \in \mathbb{N}, g \in G\right\}$ of free non commuting indeterminates and the free $F$-algebra $F\left\langle X_{G}\right\rangle$ generated by $X_{G}$. If $x_{i, g} \in X_{G}$ we say that the integer $i$ is the name of the indeterminate, and $g$ is its $G$-degree. Sometimes we shall use the word letter to point to an element of $X_{G}$ whose name and/or degree is not of specific interest. To specify that the $G$-degree of the letter $x$ is $g$ we write $\partial_{G}(x)=g$. The $G$-degree of a monomial is the sum of the $G$-degrees of the indeterminates occurring in it. This turns $F\left\langle X_{G}\right\rangle$ into a $G$-graded algebra, whose $g$-component is the linear span of all monomials of $G$-degree $g$.

If $A$ is any $G$-graded algebra, then any $G$-graded homomorphism $\varphi: F\left\langle X_{G}\right\rangle \rightarrow A$ is uniquely determined by the images $\varphi\left(x_{i, g}\right) \in A_{g}$ for all $i$ and $g$; we will often use $G$-substitution in $A$ instead of $G$-homomorphism from $F\left\langle X_{G}\right\rangle$ to $A$. Thus $F\left\langle X_{G}\right\rangle$ is in fact free in the class of $G$-graded algebras. A polynomial $f \in F\left\langle X_{G}\right\rangle$ is called a $G$-graded polynomial, and $f$ is a $G$-graded polynomial identity for $A$ if $f$ lies in the kernel of all $G$-homomorphisms $\varphi: F\left\langle X_{G}\right\rangle \rightarrow A$. The intersection of all those kernels is an ideal of $F\left\langle X_{G}\right\rangle$, stable under all $G$-endomorphisms of $F\left\langle X_{G}\right\rangle$. It is denoted by $T_{G}(A)$ and called the $T_{G}$-ideal of the graded polynomial identities of $A$. More generally, an ideal $I$ of $F\left\langle X_{G}\right\rangle$ is called a $T_{G}$-ideal if it is stable under all $G$-endomorphisms of $F\left\langle X_{G}\right\rangle$. If $\mathscr{S} \subseteq F\left\langle X_{G}\right\rangle$, the least $T_{G}$-ideal $(\mathscr{S})_{T_{G}}$ of $F\left\langle X_{G}\right\rangle$ containing $\mathscr{S}$ is called the $T_{G}$-ideal generated by $\mathscr{S}$. Any polynomial $f \in(\mathscr{S})_{T_{G}}$ is called a consequence of $\mathscr{S}$, or equivalently one says that $f$ follows from $\mathscr{S}$.

If $A$ is $G$-graded, some of its components may be trivial, that is $A_{g}=0$ for some $g \in G$. We denote $S(A):=\left\{g \in G \mid A_{g} \neq 0\right\}$, the support of $A$, and a letter $x \in X_{G}$ is supported in $A$ if $\partial_{G}(x) \in S(A)$, that is if $x$ is not a $G$-graded polynomial identity for $A$. A letter is trivial if it is not supported in $A$. We denote $\mathscr{N}_{G}(A):=\left\{x \in X_{G} \mid x \in T_{G}(A)\right\}$ the set of trivial letters of $A$. They generate a $T_{G}$-ideal of $F\left\langle X_{G}\right\rangle$ contained in $T_{G}(A)$, the ideal of trivial $G$-graded identities of $A$. As a first step in studying the graded polynomial identities of $A$ one is interested in finding a set of graded polynomials, besides those in $\mathscr{N}_{G}(A)$, generating the whole $T_{G}(A)$. 
A further step in studying the $G$-graded polynomial identities of $A$ is to obtain a more detailed description of $T_{G}(A)$, through its structure. This in general requires more specific assumptions, in particular on the base field. Here we assume that $F$ has characteristic zero, and consider graded multilinear polynomials: let $P_{n}^{G}$ denote the vector subspace of $F\left\langle X_{G}\right\rangle$ spanned by all monomials $x_{i_{1}, g_{1}} x_{i_{2}, g_{2}} \ldots x_{i_{n}, g_{n}}$ such that $\left\{i_{1}, i_{2}, \ldots, i_{n}\right\}=\{1,2, \ldots, n\}=:[n]$ and $\left(g_{1}, \ldots, g_{n}\right) \in G^{n}$. Any polynomial in $P_{n}^{G}$ is called a $G$-multilinear polynomial of degree $n$.

Since the characteristic of $F$ is zero, any $T_{G}$-ideal of $F\left\langle X_{G}\right\rangle$ is generated by the $G$-multilinear polynomials it contains, by general multilinearization process and Vandermonde arguments. More precisely, $T_{G}(A)$ is generated by the polynomials in $\bigcup_{n \in \mathbb{N}}\left(P_{n}^{G} \cap T_{G}(A)\right)$. Each $P_{n}^{G} \cap T_{G}(A)$ is actually more than a vector space. In fact, the symmetric group $S_{n}$ acts on $P_{n}^{G}$ by extending diagonally the natural action of permuting the names of the indeterminates (that is $\sigma \bullet x_{i, g}:=x_{\sigma(i), g}$ for all $\sigma \in S_{n}$ ), thus turning $P_{n}^{G}$ into a left $S_{n}$-module. Moreover, the action of $\sigma \in S_{n}$ on $X_{G}$ defines a unique $G$-automorphism of $F\left\langle X_{G}\right\rangle$, which we still denote by $\sigma$. Therefore $\sigma T_{G}(A)=T_{G}(A)$ and hence $P_{n}^{G} \cap T_{G}(A)$ is an $S_{n}$-module, whose structure is the second target aimed to. Actually, it is more convenient to describe the structure of the factor module $P_{n}^{G}(A):=P_{n}^{G} /\left(P_{n}^{G} \cap T_{G}(A)\right)$.

The $S_{n}$-structure of $P_{n}^{G}(A)$ is encoded in smaller multilinear spaces, whose description provides all necessary information on $P_{n}^{G}(A)$. In the present paper we follow the approach of [3], albeit in a different form to keep the notation as simple as possible. We quickly recall the main points of [3] within the new notation.

Given any nonempty set $\Omega$, an $\Omega$-multiset of class $k$ is any function $\mu: \Omega \rightarrow \mathbb{N}$ such that $\sum_{\omega \in \Omega} \mu(\omega)=k$. The number $\mu(\omega)$ is called the multiplicity of $\omega$ in the multiset. We shall denote the multiset by $\mu \vDash_{\Omega} k$. Each monomial $w \in P_{n}^{G}$ defines a $G$-multiset $\mu(w, \cdot) \vDash_{G} n$ : for all $g \in G, \mu(w, g)$ is the number of letters of $G$-degree $g$ occurring in $w$. Therefore the natural monomial basis of $P_{n}^{G}$ is partitioned by the $G$-multisets of class $n$. If $\alpha \vDash_{G} n$ define $V(\alpha)$ to be the vector subspace of $P_{n}^{G}$ spanned by all monomials such that $\mu(w, \cdot)=\alpha$. One has

$$
P_{n}^{G}=\bigoplus_{\alpha \models_{G} n} V(\alpha), \quad P_{n}^{G} \cap T_{G}(A)=\bigoplus_{\alpha \vDash_{G} n}\left(V(\alpha) \cap T_{G}(A)\right)
$$

and notice that both $V(\alpha)$ and $V(\alpha) \cap T_{G}(A)$ are $S_{n}$-submodules of $P_{n}^{G}$. Then (see [3], Lemma 1)

$$
\frac{P_{n}^{G}}{P_{n}^{G} \cap T_{G}(A)} \cong S_{n} \bigoplus_{\alpha \models_{G} n} \frac{V(\alpha)}{V(\alpha) \cap T_{G}(A)} .
$$

Yet a further reduction is possible. We call any function $\mathbf{t}:[n] \rightarrow G$ a $G$-degree attribution, (or simply an attribution) for self-evident reasons. For a fixed attribution $\mathbf{t}$, let $V(\mathbf{t})$ be the vector space spanned by all monomials $w \in P_{n}^{G}$ such that exactly the indeterminates $x_{1, \mathbf{t}(1)}, \ldots, x_{n, \mathbf{t}(n)}$ occur in $w$. Then $\mathbf{t}$ determines a $G$-multiset $\mu(\mathbf{t}, \cdot)$ of class $n$ by setting $\mu(\mathbf{t}, g):=\left|\mathbf{t}^{-1}(g)\right|$ for all $g \in G$. If $\alpha \vDash_{G} n$, with abuse of notation we write $\mathbf{t} \in \alpha$ if $\mu(\mathbf{t}, \cdot)=\alpha$. For a given $\alpha \vDash_{G} n$, if $\mathbf{t} \in \alpha$ then $V(\mathbf{t}) \subseteq V(\alpha) . V(\mathbf{t})$ is not an $S_{n}$-module, but is a module for a subgroup $D(\mathbf{t})$ of $S_{n}$. Precisely, since the nonempty fibers $\mathbf{t}^{-1}(g) \subseteq[n]$ form a partition of $[n]$, the subgroups $\operatorname{Sym}\left(\mathbf{t}^{-1}(g)\right)$ of $S_{n}$ mutually centralize, hence they generate a subgroup $D(\mathbf{t}) \leqslant S_{n}$, namely the direct (internal) product of the subgroups $\operatorname{Sym}\left(\mathbf{t}^{-1}(g)\right)$, hence isomorphic to the direct (external) product of the symmetric groups $S_{\alpha(g)}$. Then $V(\mathbf{t})$ is an $D(\mathbf{t})$-left module, $V(\mathbf{t}) \cap T_{G}(A)$ is a submodule and the factor 
module $V(\mathbf{t}, A)=V(\mathbf{t}) /\left(V(\mathbf{t}) \cap T_{G}(A)\right)$ is an $D(\mathbf{t})$-module as well. The main point in doing this is that ([3], Lemma 4$)$

$$
\frac{V(\alpha)}{V(\alpha) \cap T_{G}(A)} \cong_{S_{n}} F S_{n} \otimes_{F D(\mathbf{t})} \frac{V(\mathbf{t})}{V(\mathbf{t}) \cap T_{G}(A)},
$$

that is the $S_{n}$-induced module $V(\mathbf{t}, A)^{S_{n}}$. This allows the reconstruction of the $S_{n}$-structure of $P_{n}^{G}(A)$.

\section{Graded triangular Algebras}

Let $F$ be any field. In this and the following section we allow $F$ being finite. Let $H, K$ be subgroups of an abelian group $G$ and let $A, B$ be $F$-algebras graded by $H$ and $K$ respectively. We can turn the direct sum $A \oplus B$ into a $G$-graded algebra, setting

$$
(A \oplus B)_{g}:=A_{g} \oplus B_{g} \quad \forall g \in G .
$$

We assume that $T_{H}(A)$ and $T_{K}(B)$ are known, as well as $T_{G}(A \oplus B)$. In fact, $T_{G}(A)$ and $T_{G}(B)$ are easily obtained:

Lemma 1. Let $G$ be a group, $H \leqslant G$ and let $A$ be an $H$-graded $F$-algebra. If $\mathscr{S}$ is a set of polynomials such that $\mathscr{S} \cup \mathscr{N}_{H}(A)$ generates $T_{H}(A)$, then $T_{G}(A)$ is generated by $\mathscr{S} \cup \mathscr{N}_{G}(A)$.

Proof. A is turned into a $G$-graded algebra simply setting $A_{g}=0$ for all $g \in G \backslash H$. Thus $S_{G}(A)=S_{H}(A)$ (the support of $A$ is the same), and $\mathscr{N}_{G}(A) \supseteq \mathscr{N}_{H}(A)$. Let $I$ be the $T_{G}$-ideal generated by $\mathscr{S} \cup \mathscr{N}_{G}(A)$. Plainly, $I \subseteq T_{G}(A)$. Conversely, if $f \in T_{G}(A)$, split it into a sum $f=f_{H}+f^{\prime}$ where $f_{H}$ involves only letters in $X_{H}$ and each monomial of $f^{\prime}$ involves at least a letter $x \in X_{G} \backslash X_{H}$. Then clearly $f^{\prime}$ is in the $T_{G}$-ideal generated by $\mathscr{N}_{G}(A)$ (in particular, $f^{\prime} \in I$ ) and $f \in T_{G}(A)$ if and only if $f_{H} \in T_{G}(A)$. This amounts to say that $f_{H} \in T_{H}(A)$, and therefore lies in the $T_{G}$-ideal generated by $\mathscr{S} \cup \mathscr{N}_{G}(A)$. So $f \in I$, and $I=T_{G}(A)$.

Since the same arguments apply to $B$, we may assume that $T_{G}(B)$ is also known once given $T_{K}(B)$. Then it is easy to prove that $T_{G}(A \oplus B)=T_{G}(A) \cap T_{G}(B)$, and in principle this describes $T_{G}(A \oplus B)$, but in practice it is definitively far from trivial to find a set of generators for this $T_{G}$-ideal. So, we need to assume that a generating set for $T_{G}(A \oplus B)$ is explicitly known. In the rest of the paper, the $G$-support of $A \oplus B$ will be denoted simply as $S$. As an easy consequence,

Corollary 2. $S=S_{H}(A) \cup S_{K}(B), \mathscr{N}_{G}(A \oplus B)=\mathscr{N}_{G}(A) \cap \mathscr{N}_{G}(B)$.

Proof. Let $x \in X_{G}$. Then $x \in S$ if and only if $x \notin T_{G}(A \oplus B)=T_{G}(A) \cap T_{G}(B)$. Therefore either $x \notin T_{G}(A)$ or $x \notin T_{G}(B)$. Equivalently, $x \in S_{G}(A)=S_{H}(A)$ or $x \in S_{G}(B)=S_{H}(B)$.

If instead $x \in T_{G}(A \oplus B)=T_{G}(A) \cap T_{G}(B)$, then $x$ is not supported in $A$ nor in $B$, that is $x$ is a trivial letter for both $A$ and $B$.

The direct sum of graded algebras can be generalized: let $M$ be a $G$-graded ( $A, B$ )-bimodule, that is a bimodule admitting a decomposition $M=\bigoplus_{g} M_{g}$ into $F$-vector subspaces $M_{g}(g \in G)$ such that for all $h \in H, k \in K$ and $g \in G$ it holds $A_{h} M_{g} B_{k} \subseteq M_{h+g+k}$. Imposing the null multiplication on $M$ and the annihilations $B M=0=M A$ the vector space $R:=A \oplus M \oplus B$ is turned into a $G$-graded algebra, with $R_{g}=A_{g} \oplus M_{g} \oplus B_{g}$. 
A more convenient representation of $R$ is

$$
R=\left(\begin{array}{cc}
A & M \\
& B
\end{array}\right)
$$

that is identifying any element $(a, m, b) \in R$ with the matrix $\left(\begin{array}{cc}a & m \\ & b\end{array}\right)$, endowed with the usual matrix operations. This is why $R$ is called a triangular algebra.

Plainly, $S(R)=S(A \oplus B) \cup S(M)$ and $\mathscr{N}(R)=\mathscr{N}(A \oplus B) \cap \mathscr{N}(M)$. Here we assume that $S(M) \cap S(A)=\varnothing=S(M) \cap S(B)$, which means that $M$ and $A \oplus B$ have disjoint supports, and our aim is to describe the $G$-graded polynomial identities of $R$.

Let us fix the following notation:

- by $Z$ we denote the set of letters of $X_{G}$ supported in $M$;

- by $Y$ we denote the set of letters of $X_{G}$ supported in $A \oplus B$.

Hence, by our assumptions, $\{Y, Z, \mathscr{N}(R)\}$ is a partition of $X_{G}$.

\section{4. $G$-GRAded Polynomial IDEntities of $R$}

In this section we give a set of $G$-graded polynomial identities of $R$, and prove that it generates the whole $T_{G}(R)$.

Notice that of course $\mathscr{N}(R) \subseteq T_{G}(R)$ and if $z_{1}, z_{2} \in Z$ then $z_{1} z_{2} \in T_{G}(R)$ since $M^{2}=0$.

Lemma 3. For all $z_{1}, z_{2} \in Z$ and $x \in X, z_{1} x z_{2} \in T_{G}(R)$.

Proof. The statement is clear if $x \in \mathscr{N}(R) \cup Z$, so assume $x \in Y$ and let $\varphi$ : $F\left\langle X_{G}\right\rangle \rightarrow R$ be any $G$-homomorphism. Then $\varphi\left(z_{i}\right)=m_{i} \in M$ and $\varphi(x)=a+b \in$ $A \oplus B(x \in Y$ means that the letter is supported in $A \oplus B$, hence not supported in $M)$. Since $M A=B M=0_{R}$ it follows $\varphi\left(z_{1} x z_{2}\right)=0$. Hence $z_{1} x z_{2} \in T_{G}(R)$.

For any $z_{1}, z_{2} \in Z$, the polynomial $z_{1} z_{2}$ can actually be viewed as a consequence of the identities $z_{1} x z_{2}$ : indeed, it is obtained by $z_{1} x_{1,0} z_{1}$ by the specialization $x_{1,0} \rightarrow 1_{F} \in\left(F\left\langle X_{G}\right\rangle\right)_{0}$. More interesting consequences are the following:

Corollary 4. For any $z_{1}, z_{2} \in Z$ and $f \in F\left\langle X_{G}\right\rangle$, the polynomial $z_{1} f z_{2}$ is a consequence of $\left\{z_{1} x z_{2} \mid x \in X\right\}$.

Proof. Each monomial $w$ is $G$-homogeneous, therefore it can be obtained through specialization of any letter $x_{w}$ with the same $G$-degree. Hence $z_{1} w z_{2}$ follows from $z_{1} x_{w} z_{2}$. Since $f$ is the sum of its monomials, and each of them follows from $\left\{z_{1} x z_{2} \mid\right.$ $x \in X\}$, the polynomial $z_{1} f z_{2}$ follows from that set, as well.

Now let us describe the $G$-polynomial identities of $R$ involving just letters supported in $A \oplus B$ :

Lemma 5. If $f \in F\langle Y\rangle$ then $f \in T_{G}(R)$ if and only if $f \in T_{G}(A \oplus B)$.

Proof. Since $T_{G}(R) \subseteq T_{G}(A \oplus B)$, we just need to assume that $f$ belongs to $T_{G}(A \oplus$ $B)$. But since $f$ vanishes on $A \oplus B$ and just letters supported in $A \oplus B$ are involved in $f, f$ vanishes on $R$ as well.

Remark 6. There may be letters, say $y_{A}$ and $y_{B}$, supported just in $A$ and $B$ respectively. Thus both $y_{A} y_{B}$ and $y_{B} y_{A}$ are $G$-graded polynomial identities for $R$, but do not follow from $\mathscr{N}_{G}(R)$. 
Actually they are among the polynomial identities already considered: in fact $y_{A}$ not supported in $B$ means $y_{A} \in T_{G}(B)$, hence $y_{A} y_{B}, y_{B} y_{A} \in T_{G}(B)$ and, similarly, $y_{B} \in T_{G}(A)$ forces $y_{A} y_{B}, y_{B} y_{A} \in T_{G}(A)$. Thus both monomials are in $T_{G}(A) \cap$ $T_{G}(B)=T_{G}(A \oplus B)$.

Other polynomial identities are more strictly depending on the triangular structure of $R$ :

Lemma 7. For all $f \in T_{G}(A)$ and $z \in Z$, it holds $f z \in T_{G}(R)$.

Proof. Decompose $f$ as a sum $f=f_{Y}+f_{N}+f_{Z}$ where $f_{N}$ is the sum of those monomials of $f$ involving at least one trivial letter, $f_{Y}$ the sum of those monomials of $f$ involving just letters from $Y$ and let $f_{Z}$ be the sum of the remaining monomials of $f$. Notice that $f \in T_{G}(A)$ if and only if $f_{Y} \in T_{G}(A)$. In fact, $f_{Z} \in T_{G}(A)$ because each of its summands involves at least one letter from $Z$, which is not supported in $A$. The same argument works for $f_{N} \in T_{G}(A)$.

Then $f_{N} z$ and $f_{Z} z$ are consequences of $\mathscr{N}_{G}(R)$ and $\left\{z_{1} x z_{2} \mid z_{1}, z_{2} \in Z, x \in X_{G}\right\}$ respectively, so they are in $T_{G}(R)$; therefore $f z \in T_{G}(R)$ if and only if $f_{Y} z \in T_{G}(R)$. Say $f_{Y}=f_{Y}\left(y_{1}, \ldots, y_{n}\right)$. For any $G$-substitution $\varphi$, namely $\varphi\left(y_{i}\right)=a_{i}+b_{i} \in A \oplus B$ and $\varphi(z)=m \in M$, according to the $G$-degrees of $y_{1}, \ldots, y_{n}$ and $z$, it holds

$$
\varphi\left(f_{Y} z\right)=f_{Y}\left(a_{1}+b_{1}, \ldots, a_{n}+b_{n}\right) m=f_{Y}\left(a_{1}, \ldots, a_{n}\right) m+f_{Y}\left(b_{1}, \ldots, b_{n}\right) m .
$$

The first summand vanishes because $f_{Y} \in T_{G}(A)$, the second because $B M=0$. Therefore $f z \in T_{G}(R)$.

We omit the proof of the following completely analogous result

Lemma 8. For all $f \in T_{G}(B)$ and for all $z \in Z$, it holds $z f \in T_{G}(R)$.

Now consider the following graded polynomials:

- $x \in T_{G}(R)$ (the trivial letters in $\mathscr{N}_{G}(R)$ );

- $z_{1} x z_{2}\left(\right.$ for $z_{1}, z_{2} \in Z$ and $x \in X_{G}$ );

- $f \in T_{G}(A \oplus B) \cap F\langle Y\rangle$;

- $z f$ (for $z \in Z$ and $f \in T_{G}(B)$ );

- $f z$ (for $z \in Z$ and $f \in T_{G}(A)$ ).

They generate a $T_{G}$-ideal $I$ of $F\left\langle X_{G}\right\rangle$, and since each generator of $I$ belongs to $T_{G}(R)$ it holds $I \subseteq T_{G}(R)$. A last assumption is needed to prove that in fact $I \supseteq T_{G}(R)$.

Preliminary, let us recall that saying that $M$ is an $(A, B)$-bimodule amounts to say that it is a left $\left(A \otimes_{F} B^{\circ}\right)$-module, where $B^{\circ}$ is the opposite algebra of $B$. We say that $m \in M$ is an $(A, B)$-free element if its annihilator in $A \otimes_{F} B^{\circ}$ is zero.

Remark 9. An operative characterization of an $(A, B)$-free element $m \in M$ is that if $b_{1}, \ldots, b_{n} \in B$ are linearly independent and $a_{1}, \ldots, a_{n} \in A$ are all nonzero, then $\sum_{i=1}^{n} a_{i} m b_{i} \neq 0$. This is equivalent to the analogous statement obtained reversing the roles of $A$ and $B$.

Theorem 10. If any nontrivial homogeneous component of $M$ has an $(A, B)$-free element then $I=T_{G}(R)$.

Proof. Let $f \in T_{G}(R)$. We want to prove that $f \in I$. We may assume that $f$ involves only supported letters, since the trivial letters are in $I$, so $f \in F\langle Y \cup Z\rangle$. Moreover we may assume that each monomial of $f$ involves at most one letter from 
$Z$, otherwise it is in $I$. So $f$ splits in $f=f_{0}+f_{1}$, where $f_{0} \in F\langle Y\rangle$ and each monomial of $f_{1}$ involves just one letter from $Z$.

Notice that $f \in T_{G}(R)$ if and only if $f_{0}, f_{1} \in T_{G}(R)$. Indeed, if $f \in T_{G}(R)$ we may consider the $G$-endomorphism $\zeta$ of $F\left\langle X_{G}\right\rangle$ sending all letters from $Z$ in zero and fixing all other letters. Then $\zeta(f)=f_{0} \in T_{G}(R)$, so $f_{1} \in T_{G}(R)$ as well.

It is easy to prove that $f_{0} \in I$. Indeed, $f \in T_{G}(R)$ forces $f_{0} \in T_{G}(R) \cap F\langle Y\rangle \subseteq$ $T_{G}(A \oplus B) \cap F\langle Y\rangle$, hence $f_{0}$ is among the generators of $I$. Therefore we assume that $f=f_{1}$, and prove that $f \in I$.

Actually, we may assume that just a single letter $z \in Z$ occurs in $f$. Indeed, if $z_{1}, \ldots, z_{k}$ occur in $f$, then $f$ splits in $f_{z_{1}}+\cdots+f_{z_{k}}$ and, as before, $f \in T_{G}(R)$ if and only if all $f_{z_{i}}$ 's are in $T_{G}(R)$, by suitable specializations. Therefore, let $f=f\left(y_{1}, \ldots, y_{n}, z\right)$, and let

$$
f\left(y_{1}, \ldots, y_{n}, z\right)=\sum_{i} u_{i} z v_{i} \in T_{G}(R)
$$

for some polynomials $u_{i}, v_{i} \in F\langle Y\rangle$. If $u_{i} \in T_{G}(A)$ then $u_{i} z \in I$, hence we can assume that $u_{i} \notin T_{G}(A)$ for all $i$, discarding summands which are known to be in $I$. Similarly, we may assume that the $v_{i}$ are linearly independent modulo $T_{G}(B)$, because if $h \in T_{G}(B)$ then $z h \in I$. Then we proceed in proving that $f=0$ (that is, no summand is left after the mentioned reductions).

Since $u_{1} \notin T_{G}(A)$ there exists a $G$-graded substitution sending $y_{i} \rightarrow a_{i} \in A$ such that $u_{1}\left(a_{1}, \ldots, a_{n}\right) \neq 0$. Moreover, since the elements $v_{i}$ are linearly independent modulo $T_{G}(B)$ their classes $\overline{v_{i}}=v_{i}+T_{G}(B)$ in the relatively free $G$-graded algebra $F\left\langle X_{G}\right\rangle / T_{G}(B)$ are linearly independent. Hence the element $u:=\sum_{i} u_{i}\left(a_{1}, \ldots, a_{n}\right) \otimes \overline{v_{i}} \in A \otimes F\langle X\rangle / T_{G}(B)$ is nonzero. Then $u$ can be rewritten as a sum

$$
0 \neq u=\sum_{j} s_{j} \otimes \overline{g_{j}} \in A \otimes \frac{F\langle X\rangle}{T_{G}(B)}
$$

for nonzero elements $\overline{g_{j}}=g_{j}+T_{G}(B)$ and linearly independent elements $s_{j} \in A$. Since $g_{1} \notin T_{G}(B)$ there exist a $G$-substitution sending $y_{i} \rightarrow b_{i} \in B$ such that $g_{1}\left(b_{1}, \ldots, b_{n}\right) \neq 0$. Hence $r:=\sum_{j} s_{j} \otimes g_{j}\left(b_{1}, \ldots, b_{n}\right)$ is a nonzero element in $A \otimes B$. Now, since $z$ is supported in $M$, there exists an $(A, B)$-free element $m \in M$ of $G$-degree $\partial_{G}(z)$. Then consider the $G$-substitution $\varphi$ defined by $\varphi\left(y_{i}\right):=a_{i}+b_{i}$ and $\varphi(z)=m$. It is

$$
\begin{aligned}
\varphi\left(f\left(y_{1}, \ldots, y_{n}, z\right)\right) & =\sum_{i} u_{i}\left(a_{1}+b_{1}, \ldots, a_{n}+b_{n}\right) m v_{i}\left(a_{1}+b_{1}, \ldots, a_{n}+b_{n}\right) \\
& =\sum_{i} u_{i}\left(a_{1}, \ldots, a_{n}\right) m v_{i}\left(b_{1}, \ldots, b_{n}\right) \\
& =\sum_{j} s_{j} m g_{j}\left(b_{1}, \ldots, b_{n}\right) \neq 0
\end{aligned}
$$

because $m$ is free and $\sum_{j} s_{j} \otimes g_{j}\left(b_{1}, \ldots, b_{n}\right) \neq 0$.

From the proof we can extract the following easy corollary, which will be useful in the next section.

Corollary 11. If $u_{1}, \ldots, u_{k} \in F\left\langle X_{G}\right\rangle$ are not in $T_{G}(A)$ and $v_{1}, \ldots, v_{k} \in F\left\langle X_{G}\right\rangle$ are linearly independent modulo $T_{G}(B)$ then for any $z \in Z$ the polynomial $\sum_{i=1}^{k} u_{i} z v_{i}$ is not a G-polynomial identity for $R$. 


\section{5. $S_{n}$-Structure of $P_{n}^{G}(R)$}

We are now going to describe the $S_{n}$-structure of $P_{n}^{G}(R)$ according to the structures of $P_{n}^{H}(A), P_{n}^{K}(B)$ and $P_{n}^{G}(A \oplus B)$. In order to employ the representation theory of the symmetric groups, in this section the field $F$ is assumed of characteristic zero.

By [3], the problem of describing $P_{n}^{G}(R)$ is reduced to the description of the spaces $V(\alpha, R)$ for all multisets $\alpha \vDash_{G} n$ as in [3], Lemma 1.

Actually, if $\alpha(g)>0$ for some $g \notin S(R)$ then the whole $V(\alpha)$ is contained in $T_{G}(R)$ and $V(\alpha, R)$ is the null module. Hence we just need to consider multisets $\alpha \vDash_{S(R)} n$. By the way, since $S(R)=S \uplus S(M)$ (recall we denoted $S=S(A \oplus B)$ ) we may split $\alpha$ into a (pointwise) sum of an $S$-multiset $\alpha_{S}$ and an $S(M)$-multiset $\alpha_{S(M)}$. But then $\alpha_{S(M)}$ must be of class $\leqslant 1$, otherwise the whole $V(\alpha)$ lies inside $T_{G}(R)$. Therefore $\alpha_{S(M)}$ must be either zero or a Kronecker delta function $\delta_{g}$ centered at some $g \in S(M)$. In the former case, $V(\alpha, R)$ is actually the $\alpha$-component of the $S_{n}$-module $P_{n}^{G}(A \oplus B)$, which is known by assumption. Hence just the latter case has to be investigated. A little result shows that in fact it does not matter which element of $S(M)$ has been chosen.

Lemma 12. Let $g, h \in S(M)$. Then for all $\alpha_{S} \vDash_{S} n-1$ it is

$$
\frac{V\left(\alpha_{S}+\delta_{g}\right)}{V\left(\alpha_{S}+\delta_{g}\right) \cap T_{G}(R)} \cong_{S_{n}} \frac{V\left(\alpha_{S}+\delta_{h}\right)}{V\left(\alpha_{S}+\delta_{h}\right) \cap T_{G}(R)} .
$$

Proof. The map $\gamma$ switching the $g$ - and $h$-degrees $x_{i, g} \leftrightarrow x_{i, h}$ for all $i \geqslant 1$ and fixing the remaining letters is an $S_{n}$-isomorphism between $V\left(\alpha_{S}+\delta_{g}\right)$ and $V\left(\alpha_{S}+\delta_{h}\right)$.

It is also true that $V\left(\alpha_{S}+\delta_{g}\right) \cap T_{G}(R) \cong_{S_{n}} V\left(\alpha_{S}+\delta_{h}\right) \cap T_{G}(R)$. Indeed if $f \in V\left(\alpha_{S}+\delta_{g}\right) \cap T_{G}(R)$, for all $t \geqslant 1$ the sum of the monomials in $f$ involving the letter $x_{t, g}$ is also in $V\left(\alpha_{S}+\delta_{g}\right) \cap T_{G}(R)$ (by specialization), so we can harmlessly assume that just $x_{t, g}$ occurs in all monomials of $f$. Then the polynomial $\gamma(f) \in$ $V\left(\alpha_{S}+\delta_{h}\right)$ must be in $T_{G}(R)$ as well, otherwise $\gamma(f)$ must admit an expression $f=\sum_{i} u_{i} x_{t, h} v_{i}$ with $u_{i} \notin T_{G}(A)$ and $v_{i}$ linearly independent modulo $T_{G}(B)$. But then $f=\sum_{i} u_{i} x_{t, g} v_{i}$ is not in $T_{G}(R)$ by Corollary 11 .

At the light of this the study of $P_{n}^{G}(R)$ can be reduced to studying just the $S_{n-1} \times S_{1}$-modules of multilinear polynomials in which a single fixed letter $z \in Z$ occurs. Notation becomes more comfortable switching to consider $S_{n} \times S_{1}$-modules, therefore from now on we consider multisets $\alpha \vDash_{S} n$ and by $V(\alpha)\left(\subseteq P_{n+1}^{G}\right)$ we denote the vector space spanned by all monomials in which $z$ occurs with multiplicity 1 , the letters with names in $\{1, \ldots, n\}$ have $G$-degrees according to $\alpha$ and the group $S_{n} \cong S_{n} \times S_{1}$ acts on the letters fixing $z$ and renaming the other ones as usual.

If $w$ is a monomial in $V(\alpha)$ then it can be written as $w_{l} z w_{r}$ for certain submonomials $w_{l}$ and $w_{r}$. Each name $1, \ldots, n$ occurs exactly once in $w$, and $\mu(w, \cdot)=\alpha$ tells how many of them are assigned to the $G$-degree $g \in G$. Also, $\mu\left(w_{l}, \cdot\right)=: \beta$ is an $S$-multiset, telling us how many names among the $\alpha(g)$ in $w$ are placed to the left of $z$. Of course, $\beta(g) \leqslant \alpha(g)$ for all $g \in G$, and $\mu\left(w_{r}, \cdot\right)=\alpha-\beta$. Notice that the class of $\beta$ is the length of the monomial $w_{l}$.

Let us define $L=\{\beta: G \rightarrow \mathbb{N} \mid \beta \leqslant \alpha\}$. For any $\beta \in L$, let us define $V(\alpha, \beta):=\operatorname{span}_{F}\left\langle w_{l} z w_{r} \in V(\alpha) \mid \mu\left(w_{l}, \cdot\right)=\beta\right\rangle$. 
Lemma 13. For all $\alpha \vDash n$ it holds

$$
V(\alpha)=\bigoplus_{\beta \in L} V(\alpha, \beta), \quad V(\alpha) \cap T_{G}(R)=\bigoplus_{\beta \in L}\left(V(\alpha, \beta) \cap T_{G}(R)\right) .
$$

Proof. Any $w=w_{l} z w_{r} \in V(\alpha)$ lies exactly in $V\left(\alpha, \mu\left(w_{l}, \cdot\right)\right)$, and $\mu\left(w_{l}, \cdot\right) \in L$. Therefore $V(\alpha)=\bigoplus_{\beta \in L} V(\alpha, \beta)$ is clearly true. The second equality is more subtle.

Actually, the monomials of $V(\alpha)$ are parted in subsets indexed by the cartesian product $[n]^{G} \times L$. Indeed, if $w=w_{l} z w_{r}$ is a monomial in $V(\alpha)$, it determines uniquely an attribution $\mathbf{t} \in[n]^{G}$ (the indeterminates occurring in $w$ ) and a multiset $\beta \in L$ (the multiplicities of the $G$-degrees of the indeterminates to the left of $z)$. Let $W(\mathbf{t}, \beta)$ the vector subspace of $V(\alpha)$ spanned by all monomials $w_{l} z w_{r}$ in indeterminates $x_{i, \mathbf{t}(i)}(i \in[n])$ and such that $\mu_{w_{l}}=\beta$. Then actually $V(\alpha)=$ $\bigoplus_{\mathbf{t}, \beta} W(\mathbf{t}, \beta)$.

Now let $f \in V(\alpha) \cap T_{G}(R)$. Then

$$
f=\sum_{\mathbf{t} \in[n]^{G}} \sum_{\beta \in L} f_{\mathbf{t}, \beta}
$$

with $f_{\mathbf{t}, \beta} \in W(\mathbf{t}, \beta)$. We want to show that $f_{\mathbf{t}, \beta} \in T_{G}(R)$ for all $\mathbf{t}, \beta$.

For a chosen assignment $\mathbf{t} \in \alpha$, let $\zeta_{\mathbf{t}}$ be the $G$-endomorphism fixing the indeterminates $x_{i, \mathbf{t}(i)}$ and sending to 0 the other ones. Then in $\zeta_{\mathbf{t}}(f)$ just the monomials in the indeterminates $x_{i, \mathbf{t}(i)}(i \in[n])$ survive, hence $\zeta_{\mathbf{t}}(f)=\sum_{\beta \in L} f_{\mathbf{t}, \beta} \in T_{G}(R)$. Hence we may reduce our concern to the case of $f$ involving just indeterminates $x_{1, \mathbf{t}(1)}, \ldots, x_{n, \mathbf{t}(n)}$, for some $\mathbf{t}:[n] \rightarrow G$. We then want to prove that all summands of $f=\sum_{\beta \in L} f_{\beta}$ are in $T_{G}(R)$, that is each $f_{\beta}$ vanishes under any $R$-valued $G$-substitution.

Since $f$ is multilinear, we may consider only $G$-substitutions sending letters into $G$-homogeneous elements of a basis of $R$. Since each letter occurring in $f$ is in $Y$, apart from $z$, this means that each $y_{i}$ occurring in $f$ is substituted by a $G$ homogeneous basis element either of $A$ either of $B$.

Choose a multiset $\beta \in L$ and let $\varphi$ be any such $G$-substitution. For any $g \in G$ let $k(g)$ be the number of letters of $G$-degree $g$ occurring in $f$ substituted by elements of $A_{g}$ in $\varphi$. If there exists $g \in G$ such that $\beta(g) \neq k(g)$ then either $\beta(g)<k(g)$ and at least one element of $A_{g}$ is placed to the right of $\varphi(z)$ in each summand of $\varphi\left(f_{\beta}\right)$, either $\beta(g)>k(g)$, so at least an element of $B$ occurs to the left of $\varphi(z)$ in each summand of $\varphi\left(f_{\beta}\right)$. In both cases, $\varphi\left(f_{\beta}\right)=0$.

Therefore assume that $k(g)=\beta(g)$ for all $g \in G$. Then $\varphi\left(f_{\beta^{\prime}}\right)=0$ for all $\beta^{\prime} \neq \beta$. But, since $f \in T_{G}(R)$, we get $0=\varphi(f)=\varphi\left(f_{\beta}\right)$ and $\varphi\left(f_{\beta}\right)=0$, after all.

This proves that all components $f_{\beta}$ (actually, all $f_{\mathbf{t}, \beta}$ ) of $f$ are in for $T_{G}(R)$ as well.

Notice that for all $\beta \leqslant \alpha$ the space $V(\alpha, \beta)$ is an $S_{n}$-modules of $V(\alpha)$. Since $V(\alpha) \cap T_{G}(R)=\bigoplus_{\beta \in L}\left(V(\alpha, \beta) \cap T_{G}(R)\right)$, we get the consequence

Corollary 14. For all $\alpha \vDash_{G} n$ it holds

$$
V(\alpha, R) \cong_{S_{n}} \bigoplus_{\beta \in L} \frac{V(\alpha, \beta)}{V(\alpha, \beta) \cap T_{G}(R)}=: \bigoplus_{\beta \in L} V(\alpha, \beta, R) .
$$

Therefore the $S_{n}$-structure of $V(\alpha, R)$ is determined from the $S_{n}$-structure of $V(\alpha, \beta, R)$ for all $\beta \in L$, hence we need a finer description of the spaces $V(\alpha, \beta)$. 
Let $\beta \leqslant \alpha \vDash_{S} n$. Each monomial $w=w_{l} z w_{r} \in V(\alpha, \beta)$ uniquely determines an ordered pair $(J, \mathbf{t})$ where $J$ is the subset of $[n]$ consisting of the names of the letters occurring in $w_{l}$, and $\mathbf{t}$ is the attribution function of $w$. Notice that $|J|$ is the class of $\beta, \mathbf{t} \in \alpha$ and the restriction $\mathbf{t}_{J}$ to $J$ belongs to $\beta$, that is $\mathbf{t}_{J} \in \beta$.

Let $\Omega(\alpha, \beta)$ be the set of all such pairs.

Lemma 15. $S_{n}$ acts on $\Omega(\alpha, \beta)$. More precisely, if $(J, \mathbf{t}),\left(J^{\prime}, \mathbf{t}^{\prime}\right) \in \Omega$, there exists $\sigma \in S_{n} \operatorname{such}$ that $\left(J^{\prime}, \mathbf{t}^{\prime}\right)=\left(\sigma J, \mathbf{t} \sigma^{-1}\right)$.

Proof. If $\sigma \in S_{n}$ then $\left(\sigma J, \mathbf{t} \sigma^{-1}\right) \in \Omega(\alpha, \beta)$. Indeed, if $w=w_{l} z w_{r} \in V(\alpha, \beta)$ is a monomial defining $(J, \mathbf{t})$, then $\sigma w \in V(\alpha, \beta)$ defines $\left(\sigma J, \mathbf{t} \sigma^{-1}\right)$. It follows immediately that $S_{n}$ acts on $\Omega(\alpha, \beta)$.

In order to show that the action is transitive, notice that by assumption for any $g \in G$ the fibers of $g$ in $\mathbf{t}_{J}$ and in $\mathbf{t}_{J^{\prime}}^{\prime}$ are equal to $\beta(g)$. Let $\lambda_{g}$ be any bijection among them. Repeating this step for each $g \in S$, we get a bijection $\lambda=\prod_{g \in S} \lambda_{g}$ such that $\lambda J=J^{\prime}$. Similar steps can be repeated to build a bijection $\rho$ from $[n] \backslash J$ to $[n] \backslash J^{\prime}$. Then set $\sigma=\lambda \rho$. Clearly, $\sigma \in S_{n}$, and $\sigma J=J^{\prime}$.

It is straightforward to check that $\mathbf{t}^{\prime}=\mathbf{t} \sigma^{-1}$.

For $(J, \mathbf{t}) \in \Omega(\alpha, \beta)$ let $V(J, \mathbf{t})$ be the vector subspace spanned by the monomials of $V(\alpha, \beta)$ defining $(J, \mathbf{t})$. We record the following easy consequence of the preceding Lemma:

Corollary 16. For all pairs $(J, \mathbf{t}) \in \Omega(\alpha, \beta)$, the vector spaces $V(J, \mathbf{t})$ are pairwise isomorphic, as well as the subspaces $V(J, \mathbf{t}) \cap T_{G}(R)$ and the factor spaces $V(J, \mathbf{t}) /\left(V(J, \mathbf{t}) \cap T_{G}(R)\right)$.

Proof. Fix $(J, \mathbf{t}) \in \Omega(\alpha, \beta)$. Then $\Omega(\alpha, \beta)=\left\{\left(\sigma J, \mathbf{t} \sigma^{-1}\right) \mid \sigma \in S_{n}\right\}$, and $\sigma V(J, \mathbf{t})=$ $V\left(\sigma J, \mathbf{t} \sigma^{-1}\right)$. Now just recall that $\sigma$ is a $G$-automorphism of $F\left\langle X_{G}\right\rangle$.

If $\beta$ has class $k$, let us choose $J=\{1, \ldots, k\}$, and fix any attribution $\mathbf{t}$ such that $(J, \mathbf{t}) \in \Omega(\alpha, \beta)$. Define

$$
N:=V([k], \mathbf{t}), \quad N(R):=\frac{N}{N \cap T_{G}(R)}, \quad d_{N}:=\operatorname{dim}_{F} N(R) .
$$

We should emphasize that $N$ depends on $\alpha$ and $\beta$, as well as on the chosen $\mathbf{t}$. By the way, since our concern is to describe the structure of $V(\alpha, \beta, R)$ for fixed $\alpha$ and $\beta$, we keep the notation to the very essentials.

Thus $N$ is chosen as a representative for the isomorphism class of the vector spaces corresponding to the pairs in $\Omega(\alpha, \beta)$, and $N(R)$ of the factor spaces.

Similarly to what happens in a general setup, we are now going to define a suitable subgroup $D=D(\mathbf{t})$ of $S_{n}$ turning $N$ into a left $D$-module.

Let us denote by $\mathbf{t}_{l}$ the restriction of $\mathbf{t}$ to the set $[k]$, and by $\mathbf{t}_{r}$ the restriction to $\{k+1, \ldots, n\}$. The nonempty fibers of $\mathbf{t}_{l}$ define a subgroup of $S_{k}$, namely the direct product $D_{l}$ of the subgroups $\operatorname{Sym}\left(\mathbf{t}_{l}^{-1}(g)\right)$ for $g \in G$. Similarly, the nonempty fibers of $\mathbf{t}_{r}$ define a subgroup $D_{r}$ of $\operatorname{Sym}(\{k+1, \ldots, n\})$. Notice that that $D_{l}$ and $D_{r}$ mutually centralize in $S_{n}$, and set $D:=D_{l} \times D_{r}$.

The vector space $N$ is thus turned into a left $D$-module, and $N \cap T_{G}(R)$ is a submodule; the factor space $N(R)$ inherits the $D$-module structure. We can now prove the main result of this section: 


\section{Theorem 17.}

$$
\frac{V(\alpha, \beta)}{V(\alpha, \beta) \cap T_{G}(R)} \cong_{S_{n}} F S_{n} \otimes_{F D} N(R)
$$

Proof. Let us define

$$
\psi: \begin{array}{rll}
F S_{n} \times \frac{N}{N \cap T_{G}(R)} & \rightarrow & \frac{V(\alpha, \beta)}{V(\alpha, \beta) \cap T_{G}(R)} \\
\left(\sigma, f+\left(N \cap T_{G}(R)\right)\right) & \rightarrow & \sigma f+\left(V(\alpha, \beta) \cap T_{G}(R)\right)
\end{array}
$$

The function $\psi$ is well defined, because if $f \in N$ then $\sigma f \in V(\alpha, \beta)$; if moreover $f \in N \cap T_{G}(R)$ then $\sigma f \in V(\alpha, \beta) \cap T_{G}(R)$ because $\sigma$ is (can be extended uniquely to) a $G$-automorphism of $F\left\langle X_{G}\right\rangle$, so $\sigma T_{G}(R)=T_{G}(R)$.

Checking that $\psi$ is $F D$-balanced is immediate, hence it defines uniquely an $S_{n^{-}}$ module homomorphism $\psi^{*}: F S_{n} \otimes_{F D} N(R) \rightarrow V(\alpha, \beta, R)$. Notice that

$$
\operatorname{dim}\left(F S_{n} \otimes_{F D} V(R)\right)=\left(\begin{array}{l}
n \\
k
\end{array}\right)\left(\begin{array}{l}
k \\
\beta
\end{array}\right)\left(\begin{array}{c}
n-k \\
\alpha-\beta
\end{array}\right) d_{N}=\operatorname{dim} N(R),
$$

where $\left(\begin{array}{l}k \\ \beta\end{array}\right)$ (or $\left(\begin{array}{c}n-k \\ \alpha-\beta\end{array}\right)$, respectively) denotes the multinomial coefficient corresponding to the multiset $\beta$ of class $k$ (to the multiset $\alpha-\beta$ of class $n-k$, respectively). Therefore the spaces have the same dimension. Since $\psi^{*}$ is plainly surjective, this is enough to conclude that $\psi^{*}$ is indeed an $S_{n}$-isomorphism.

The $D_{l} \times D_{r}$-structure of $N(R)$ is related to the structures of $P_{n}^{G}(A)$ and $P_{n}^{G}(B)$. More precisely, we get our second result of this section:

\section{Proposition 18.}

$$
N(R) \cong D_{l} \times D_{r} V\left(\mathbf{t}_{l}, A\right) \otimes_{F} V\left(\mathbf{t}_{r}, B\right) .
$$

Proof. Recall that $V\left(\mathbf{t}_{l}, A\right)=\frac{V\left(\mathbf{t}_{l}\right)}{V\left(\mathbf{t}_{l}\right) \cap T_{G}(A)}$ is a left $D_{l}$-module, and similarly $V\left(\mathbf{t}_{r}, B\right)$ is a left $D_{r}$-module. Define

$$
\begin{array}{clc}
\varphi: V\left(\mathbf{t}_{l}, A\right) \times V\left(\mathbf{t}_{r}, B\right) & \rightarrow & N(R) \\
(\bar{f}, \bar{g}) & \longrightarrow & f z g+\left(N \cap T_{G}(R)\right)
\end{array}
$$

$\varphi$ is well defined: if $(f, g) \in V\left(\mathbf{t}_{l}\right) \times V\left(\mathbf{t}_{r}\right)$ then $f z g \in V(\alpha, \beta)$; moreover, if $f \in$ $V\left(\mathbf{t}_{l}\right) \cap T_{G}(A)$ then $f z g \in T_{G}(R)$ because $f z \in T_{G}(R)$. The same happens when $g \in T_{G}(B)$. It is clear that $\varphi$ is $F$-linear and commutes with the $D_{l} \times D_{t}$-action, so $\varphi$ uniquely defines an homomorphism $\varphi^{*}: V\left(\mathbf{t}_{l}, A\right) \otimes_{F} V\left(\mathbf{t}_{r}, B\right) \rightarrow N(R)$ of left $D_{l} \times D_{r}$-modules. We prove that $\varphi^{*}$ is an isomorphism by producing its inverse.

Define at first

$$
\begin{aligned}
& \psi: \quad N \quad \rightarrow \quad V\left(\mathbf{t}_{l}, A\right) \otimes V\left(\mathbf{t}_{r}, B\right) . \\
& w_{l} z w_{r} \quad \longrightarrow \quad \overline{w_{l}} \otimes \overline{w_{r}}
\end{aligned}
$$

This function is plainly a surjective homomorphism of $H_{l} \times H_{r}$-modules. We prove that $N \cap T_{G}(R) \subseteq \operatorname{ker} \psi$.

So assume on the contrary that there is some $f \in N \cap T_{G}(R)$ such that $\psi(f) \neq$ 0 . Since $f \in N$ it can be written $f=\sum_{i=1}^{m} u_{i} z v_{i}$ for certain polynomials $u_{i}=$ $u_{i}\left(x_{1, \mathbf{t}(1)}, \ldots, x_{k, \mathbf{t}(k)}\right), v_{i}=v_{i}\left(x_{k+1, \mathbf{t}(k+1)}, \ldots, x_{n, \mathbf{t}(n)}\right)$ and a minimal $m$.

By minimality, none of the elements among the $u_{i}$ 's belongs to $T_{G}(A)$. For the same reason, the $v_{i}$ 's constitute a linearly independent set modulo $T_{G}(B)$.

But then, by Corollary 11, $f \notin T_{G}(R)$, a contradiction.

Therefore the map $\bar{\psi}: N(R) \rightarrow V\left(\mathbf{t}_{l}, A\right) \otimes V\left(\mathbf{t}_{r}, B\right)$ sending $\bar{f} \rightarrow \psi(f)$ is a well defined $D_{l} \times D_{r}$-module homomorphism. It is immediate to check that $\varphi^{*}$ and $\bar{\psi}$ are inverse functions. 
Typically, if the graded polynomial identities of $A$ and $B$ are known, their structure is given through $P_{n}^{H}(A)$ and $P_{n}^{K}(B)$ for all $n \in \mathbb{N}$. Thus the structure of $P_{n}^{G}(A)$ (and of $\left.P_{g}^{G}(B)\right)$ is known.

Indeed, $P_{n}^{H}(A)$ is the direct sum of the submodules $V(\alpha, A) \cong(V(\mathbf{t}, A))^{S_{n}}$ where $\mathbf{t}$ is any attribution in $\alpha \vDash_{H} n$. The same holds for $P_{n}^{G}(A)$, so the only difference may be in $\alpha \vDash_{G} n$ instead of $\alpha \vDash_{H} n$. By the way the only attributions giving possibly non trivial summands $V(\alpha, A)$ correspond to $\alpha \vDash_{S_{H}(A)} n$ or $\alpha \vDash_{S_{G}(A)} n$ respectively, and since $S_{H}(A)=S_{G}(A)$ they are exactly the same. More explicitly, if $\mathbf{t}:[n] \rightarrow H$ is an attribution such that $V(\mathbf{t}, A) \neq 0$, then actually $\mathbf{t}([n]) \subseteq$ $S_{H}(A)=S_{G}(A)$ and therefore $V(\mathbf{t}, A)^{S_{n}}$ participates just the same in both $P_{n}^{H}(\bar{A})$ and $P_{n}^{G}(A)$.

Now let us come to the point: we can combine the previous main results and get a simple and practical way of describing the structure of the $G$-graded polynomial identities of $R$ by means of the Littlewood-Richardson rule. This is the sense of the following

\section{Corollary 19.}

$$
V(\alpha, \beta, R) \cong_{S_{n}}(V(\beta, A) \otimes V(\alpha-\beta, B))^{S_{n}} .
$$

Proof. It is $D_{l} \times D_{r} \leqslant S_{k} \times S_{n-k}$ (we are actually identifying $S_{n-k}$ with $\operatorname{Sym}(\{k+$ $1, \ldots, n-k\}))$. Since $N(R) \cong{ }_{D_{l} \times D_{r}} V\left(\mathbf{t}_{l}, A\right) \otimes V\left(\mathbf{t}_{r}, B\right)$, by inducing to $S_{k} \times S_{n-k}$ we get

$$
N(R)^{S_{k} \times S_{n-k}} \cong_{S_{k} \times S_{n-k}} V\left(\mathbf{t}_{l}, A\right)^{S_{k}} \otimes V\left(\mathbf{t}_{r}, B\right)^{S_{n-k}} .
$$

We know that $V\left(\mathbf{t}_{l}, A\right)^{S_{k}} \cong_{S_{k}} V(\beta, A), V\left(\mathbf{t}_{r}, B\right)^{S_{n-k}} \cong_{S_{n-k}} V(\alpha-\beta, B)$. Inducing once more from $S_{k} \times S_{n-k}$ to $S_{n}$ we get

$$
\left(N(R)^{S_{k} \times S_{n-k}}\right)^{S_{n}} \cong S_{n}(V(\beta, A) \otimes V(\alpha-\beta, B))^{S_{n}},
$$

so by transitivity

$$
\left(N(R)^{S_{k} \times S_{n-k}}\right)^{S_{n}} \cong_{S_{n}} N(R)^{S_{n}} \cong_{S_{n}} V(\alpha, \beta, R) .
$$

\section{ACKNOWLEDGEMENT}

We wish to thank the referee for his/her useful remarks and sensible comments.

\section{REFERENCES}

[1] E. Aljadeff, A. Kanel-Belov, Representability and Specht problem for G-graded algebras, Adv. Math. 225 (5) (2010), 2391-2428

[2] E. Aljadeff, A. Giambruno, D. La Mattina, Graded polynomial identities and exponential growth, J. Reine Angew. Math. 650 (2011), 83-100.

[3] O. M. Di Vincenzo, Cocharacters of G-graded Algebras, Comm. Algebra 24 (10) (1996), 3293-3310.

[4] Di Vincenzo, Drensky, The basis of the graded polynomial identities for superalgebras of triangular matrices, Comm. Algebra 24 (2) (1996), 727-735.

[5] O. M. Di Vincenzo, R. La Scala, Block-triangular matrix algebras and factorable ideals of graded polynomial identities, J. Algebra 279 (2004), 260-279.

[6] O. M. Di Vincenzo, V. Nardozza, $\mathbb{Z}_{2}$-graded cocharacters for superalgebras of triangular matrices, J. Pure and Appl. Algebra 194 (2004), 193-211.

[7] A. Giambruno, M. Zaicev, Polynomial Identities and Asymptotic Methods, Math. Surveys and Monographs Vol. 122 (2005) - AMS

[8] A. Giambruno, M. Zaicev, Minimal Varieties of Algebras of exponential growth, Adv. Math. 174 (2003), 310-323. 
[9] A. Giambruno, M. Zaicev, Codimensions Growth and Minimal Superalgebras, Trans. Amer. Math. Soc. 355 (12) (2003), 5091-5117.

[10] A. R. Kemer, Varieties and $\mathbb{Z}_{2}$-graded algebras, Izv. Akad. Nauk SSSR, Ser. Mat. 48 (1984), 1042-1059. Translation: Math. USSR Izv. 25 (1985), 359-374.

[11] T. Y. Lam, A First Course in Noncommutative Rings, Graduate Texts in Mathematics 131, Second Edition (2001), Springer-Verlag.

[12] J. Lewin, A Matrix Representation for Associative Algebras, I, Trans. Amer. Math. Soc. 188 (1974), 293-308

[13] I. Sviridova, Identities of pi-algebras graded by a finite abelian group, Comm. Algebra 39 (2011), 3462-3490.

[14] A. Valenti, The graded polynomial identities of upper triangular matrices of order two, J. Pure and Appl. Algebra 172 (2002), 325-335.

Dipartimento di Matematica, Informatica ed Economia, Università degli Studi della Basilicata, viale dell'Ateneo lucano 10, 85100 Potenza, Italia

Email address: onofrio.divincenzo@unibas.it

Dipartimento di Matematica, Università degli Studi di Bari, via Orabona 4, 70125 BARI, ItALIA

Email address: $₫$ vincenzo.nardozza@uniba.it 